\title{
La percepción del factor de los padres en el aprendizaje del inglés en la escuela primaria: un estudio empírico
}

\author{
Elena Carolina Hewitt \\ Departamento de Filología Inglesa, Universidad de Granada
}

Recibido: 20 octubre 2006 / Aceptado: 30 octubre 2006

ISSN: $1697-7467$

\begin{abstract}
RESUMEN: Este artículo presenta un estudio exploratorio de 42 niños que comenzaron su aprendizaje del inglés (edad media 8.25 años) basándonos en datos sobre el conocimiento del inglés de sus padres y - la ayuda que éstos aportaron al inglés que aprendían los alumnos. Durante un año académico, los alumnos completaron cuatro pruebas de inglés escrito, cuatro pruebas auditivas y un cuestionario sobre sus antecedentes y los de sus familias. Los datos de esta percibida influencia paterna y materna conseguidos a través del cuestionario se analizaron junto con el rendimiento académico, utilizando para ello un análisis de MANOVA (Múltiple Análisis de Varianza). Adicionalmente, se somete a debate la posibilidad de que la opinión que tienen los profesores sobre los verdaderos logros en el inglés de sus alumnos puede estar algo deformada a causa de esta ayuda de los padres. La importancia de esto es que no sólo tiene consecuencias para estos niños, sino especialmente para aquellos alumnos que no reciben ninguna ayuda paterna. Esto se debe a que los alumnos que aparentemente progresan menos con el inglés pueden, en realidad, ser mejores que los otros ya que están progresando solos, sin la guía paterna y por mérito propio.

Debido a estos hallazgos, se recomienda que los profesores averigüen tanta información como sea posible de sus alumnos sobre la ayuda o falta de ayuda que reciben de sus padres. Para ese fin, se adjunta el cuestionario ya pilotado y experimentado y luego utilizado en este estudio. Así, los profesores pueden formarse una idea más cierta de la Palabras clave: La ayuda de la madre y del padre en inglés; el rendimiento académico; MANOVA (Múltiple Análisis de Varianza).
\end{abstract}

\begin{abstract}
This paper describes an exploratory study in which 42 children took part and where data was collected on the mothers' and fathers' help and knowledge of English as a foreign language. This data was then analysed together with the children's marks on an English test which was given four times throughout the school year. The design of the study was therefore one of Repeated Measures and the statistical analysis was of MANOVA (Simple Multiple Analysis of variance) and a Repeated Measures MANOVA. The results are discussed. Additionally therefore, we look at the possibility that teachers' judgments of their pupils may not be quite accurate because of help from the mothers and fathers. This may not only have consequences for the good students but mostly for the ones apparently not doing as well as the former. Although they have no parental help, in fact they are progressing on their own independent aptitude and have more merit. We therefore suggest that teachers collect as much data as possible about
\end{abstract}


their pupils. To aid teachers in this we have included the questionnaire used in this study in the appendix for photocopying.

Key Words: Mother's and father's help with English, academic achievement, MANOVA (Multiple Analysis of Variance).

\section{INTRODUCCIÓN}

El conjunto específico de variables sobre la influencia y ayuda de los padres en niños aprendiendo inglés como idioma extranjero parece que no se ha investigado anteriormente de forma empírica. Burstall (1975) es uno de los pocos trabajos empíricos que han estudiado la influencia de los padres en el aprendizaje de la lengua extranjera (LE). Sin embargo, esta autora descubrió las áreas específicas donde los progenitores eran importantes con el francés. En la mencionada investigación, Burstall (1975) encontró que el ambiente doméstico afecta a la motivación y por tanto al rendimiento académico. Así mismo, el estudio clásico de Gardner y Smythe (1975) descubrió que la motivación de los alumnos se regula mediante la influencia paterna.

En el campo de la psicología de la educación, las variables de la familia se han explorado con más profundidad y se considera que son fundamentales. Sin embargo, según Benson (1980), esta conexión tan robusta entre antecedentes de familia y el rendimiento académico no se ha utilizado mucho para la mejora de la práctica educativa. Tampoco lo han empleado para el bien de las familias o para el de las autoridades educativas.

Marjoribanks (1984) llegó a la conclusión de que un aumento en el apoyo materno se asocia con un alza en la escala de ambiciones académicas del alumno. Si la ocupación paterna era de la clase obrera, ninguna asociación se encontró entre este apoyo y las ambiciones de los alumnos hasta que se alcance en la escala una determinada puntuación. La variable del apoyo paterno percibido, por lo tanto, tuvo una relación curvilínea con las ambiciones académicas. La intensidad de esta última varió según la condición ocupacional paterna. La mayoría de los datos obtenidos por Olneck y Bills (1980), se refieren al padre. Un efecto importante para el nivel académico de alumnos se relaciona con los padres que tienen estudios universitarios, trabajo del tipo denominado «cuello blanco» (no manual) o con menor número de miembros en la familia. Cano García (1995) encontró que los padres con bajos ingresos no preguntaban a sus hijos por sus estudios, ni estos niños consultaban con sus padres.

Christenson (1990) describe la reciente tendencia en el campo de la investigación de la influencia de los padres. Su estudio parece dejar atrás la simple relación de la condición socio-económica de los padres a favor de un proceso más delicado interpersonal e intrafamiliar con resultados más específicos para los niños. Madrid y Manrique (1983) también han demostrado la influencia de la familia en el lenguaje infantil.

Con respecto a la investigación descrita por Elley (1994), el proyecto extensivo del IEA (International Evaluation and Assessment) contaba con más de 30 países. Entre 1989 y 1992 inspeccionaron, entre otras cosas, las relaciones entre la condición socio-económica de la familia junto con la ayuda paterna, el rendimiento académico (lectura y escritura) en la escuela primaria y la lengua materna. La relación entre la condición socio-económica y el 
nivel académico no era lineal, sino de tipo "gaussiano" (siguiendo la distribución que marca la campana de Gauss). Por otra parte, la relación entre el nivel académico del padre y el nivel de lectura y escritura de sus hijos fue lineal.

Los resultados del aún más reciente estudio IEA del año 2003 están disponibles en Internet. El correspondiente informe de Mullis, Martin, Gonzalez y Kennedy (2003) muestra los datos de este estudio PIRLS (Progress in International Reading Literacy), una réplica del estudio anterior del año 1991. Esta vez participaron 35 países en un estudio efectuado con niños de 10 años de edad (quinto curso). La información también se recogió de los niños directamente, al igual que en el estudio empírico aquí presentado. Los resultados mostraron que, con respecto a la ocupación de los padres, la capacidad de lectura era mayor cuando ambos padres trabajaban a tiempo completo y peor cuando ambos trabajaban a tiempo parcial. Esta capacidad cambiaba paulatinamente con relación a los profesionales, con la capacidad más alta de lectura en estudiantes con padres cualificados para sus ocupaciones profesionales y una capacidad baja para hijos con padres de trabajos manuales y con una madre que nunca había hecho trabajos remunerados.

Con respecto al papel de la madre, Stevenson y Baker (1987) descubrieron que hay relación entre el nivel de educación de la madre y las notas de sus hijos. Cano García (1995) encontró que la influencia de la ayuda de la madre sobre el rendimiento académico es pequeña. Sin embargo, el análisis daba una cifra de estadística negativa que posiblemente se puede deber a que las madres ayudan a aquellos niños que tienen más problemas.

En la parte más descriptiva del informe de Burstall del año 1970 (páginas 45 a 46) se describen las actitudes de los niños en el aprendizaje del francés y la influencia que los éstos recibieron de sus padres en este proceso. Según las observaciones, parece ser la madre la que más ánimo da a sus hijos en el aprendizaje de la lengua, ya que esos niños lograron un nivel más alto de dominio del idioma.

Asímismo, West, Noden y Edge (1998) comentaron que era la madre la que generalmente se hacía cargo de supervisar los deberes escolares de sus hijos en casa. La madre ayudaba con mayor frecuencia en las tareas escolares, a excepción de las matemáticas en las que era el padre el que solía echar una mano. Los mismos autores también descubrieron diferencias importantes en la relación entre el nivel educativo de la madre y el empleo de cuadernos de ejercicios o tutores particulares. Cuanto más alto es el nivel educativo de la madre, mayor es el uso que hace de estos recursos educativos. West, Noden y Edge (1998) recomendaron medir el nivel educativo de la madre en estudios de investigación de este tipo ya que aporta más beneficios que la tradicional codificación de su clase social. Mueller y Parcel (1981) consideraron como importante utilizar el nivel ocupacional de la madre como una medida mejor y global.

La participación de los padres se puede medir a través de ellos mismos o a través de la percepción de sus hijos. Ambas maneras son igualmente respetadas y tienen larga tradición. Entre los investigadores que han utilizado la percepción que los hijos tienen de la participación de sus padres están Keith, Keith, Troutman, Bickley, Trivette y Singh, (1993) o Grolnick, Ryan y Deci (1991).

En el campo del inglés como segundo idioma (ESL) parece ser que se están llevando a cabo muchos programas que incorporan la influencia de los padres en las escuelas, pero existe poca investigación al respecto. Uno de los pocos estudios que informan sobre la investigación o los hallazgos en el campo del inglés como segundo idioma (ESL) es Henderson 
(1987) con su bibliografía anotada. Cita 49 estudios que reflejan los efectos de la participación de los padres en el rendimiento académico de los niños. Río Heller y Fantuzzo (1993) incorporaron con éxito en sus estudios diferentes maneras de participación de los padres. Estas son: a) los padres daban recompensas según el grado de desempeño académico, b) los padres asistían a sesiones en el aula para observar a sus niños tomando parte en el aprendizaje cooperativo y, c) los padres hacían de profesores ayudantes en el aula además de ponerse en contacto con otros padres que tenían interés en el proyecto. No se han encontrado estudios que incluyan la recogida sistemática de datos, por parte de las escuelas, sobre el conocimiento paterno y/o la ayuda a los alumnos con el inglés u otro idioma extranjero.

\section{MÉTodo}

\subsection{Hipótesis}

En el estudio que se describe a continuación, la primera hipótesis es que hay diferencia significativa en el rendimiento académico entre los alumnos que perciben que sus padres tienen conocimiento del inglés y los que no. En segundo lugar, partimos de que hay también diferencia significativa entre los alumnos que perciben la ayuda de los padres y los que no. Y por último, pensamos que existe una tercera diferencia significativa entre los alumnos que perciben que sus padres les han ayudado un mayor número de veces. En cada caso, el nivel alfa es $p \leq 0.05$.

\subsection{Muestra}

La muestra empleada fue de 42 niños de ambos sexos, 22 niñas y 20 niños. Esta era la población entera del tercer curso de un colegio público de Andalucía. Los participantes eran de aproximadamente ocho años de edad (edad media 8.25 años), es decir, la edad estipulada localmente para comenzar un idioma extranjero en la escuela. Los alumnos vienen de familias de clase socio-económica media o baja.

\subsection{Instrumentos}

\subsubsection{Cuestionario}

Se utilizó el cuestionario del apéndice 1 para recoger datos de los alumnos para las variables independientes. Estas últimas fueron: el Percibido conocimiento y número de veces que fueron ayudados con el inglés (por el padre y la madre). En nuestro estudio, el apoyo paterno era el que percibían los alumnos.

El cuestionario fue diseñado por la autora de este trabajo y anteriormente pilotado con una población parecida. Los participantes del estudio piloto eran de la misma edad, clase socio-económica y entorno que la muestra de este estudio.

Únicamente nos conciernen de momento, las preguntas del cuestionario específicamente 
relacionadas con la influencia de la familia. Las preguntas exactas eran, como se indica a continuación y pueden encontrarse en el cuestionario adjunto, las preguntas números 10 a 15 . La redacción final de las preguntas sobre los padres tuvo en cuenta la corta edad de los alumnos, así como los resultados del estudio piloto que mostraron que estas preguntas eran las más indicadas para recoger la información deseada. Había un total de seis variables independientes de familia:

a) El Percibido conocimiento del inglés del padre,

b) La Percibida ayuda dada por el padre,

c) El Percibido número de veces ayudado por el padre,

d) El Percibido conocimiento del inglés de la madre,

e) La Percibida ayuda dada por la madre,

f) El número de veces que ayudó la madre.

\subsubsection{Las pruebas de inglés}

Las variables dependientes se controlaron con la prueba de inglés de Abbs, Worrall y Ward (1993). Esta prueba está compuesta de dos de partes, una escrita y una prueba auditiva. Las cifras "inter-rater" de fiabilidad fueron altas, los coeficientes Pearson de correlación (r) fueron .97 y .98 para la prueba auditiva y escrita respectivamente.

\subsection{Procedimiento}

La profesora dio el cuestionario a los alumnos durante la primera clase. Ellos lo completaron de forma individual con el profesor cerca para resolver cualquier duda. La misma profesora dio la prueba de inglés en cuatro ocasiones distanciadas a lo largo del año escolar. Los intervalos entre las pruebas fueron por lo menos de dos meses.

\subsection{Análisis Estadístico}

A fin de ver si los alumnos que recibieron ayuda paterna sacaron notas estadísticamente más altas que aquellos que no tuvieron ayuda, se sometieren los resultados de las prueba a los análisis siguientes. El MANOVA simple (Análisis Múltiple de Varianza) que analiza estadísticas múltiples y es empleado para descubrir una diferencia significativa entre dos o más cifras de media. Este análisis se efectúa con múltiples variables dependientes y es especialmente útil aquí, ya que se realizó más de una prueba de inglés. El MANOVA permite poner todas estas variables en el mismo análisis a la vez para ver si la nota es significativa en conjunto. Además, se aplicó un análisis de Medidas Repetidas de MANOVA a fin de obtener información complementaria/adicional. La misma prueba auditiva y escrita se usó repetidamente a lo largo del curso académico. Esto permitía un diseño de investigación de tipo medidas repetidas y su correspondiente análisis en cada una de las pruebas al contrario del MANOVA simple. En cada caso, el nivel alfa fue $p$ $\leq 0.05$. 


\section{Resultados}

\subsection{Variable 1: El Percibido conocimiento del inglés del padre (tabla 1)}

El análisis de Medidas Repetidas MANOVA con las pruebas auditivas 1-4, y la variable del Percibido conocimiento del inglés del padre mostró un efecto principal significativo. El MANOVA simple y la variable del Percibido conocimiento del inglés del padre mostró un efecto principal significativo con las pruebas auditivas 1-4.

El análisis de Medidas Repetidas MANOVA (también presentado en la tabla 1) con las pruebas escritas (1-4) y la variable del Percibido conocimiento del inglés del padre mostró un efecto principal significativo. El análisis MANOVA simple y la variable del Percibido conocimiento del inglés del padre mostró un efecto principal significativo con las pruebas escritas 1-4. También era significativo en todas las F Univariadas excepto la prueba primera.

Tabla 1: Percibido conocimiento del inglés del padre: las estadísticas para las pruebas escritas y auditivas

\begin{tabular}{|c|c|c|c|c|c|}
\hline \multicolumn{3}{|c|}{$\begin{array}{l}\text { MANOVA de Medidas Repetidas en Pruebas } \\
\text { auditivas + Percibido conocimiento del inglés del padre }\end{array}$} & \multicolumn{3}{|c|}{$\begin{array}{l}\text { MANOVA de Medidas Repetidas en Pruebas } \\
\text { escritas + Percibido conocimiento del inglés del } \\
\text { padre }\end{array}$} \\
\hline \multicolumn{3}{|l|}{ Efecto principal } & \multicolumn{3}{|l|}{ Efecto principal } \\
\hline $\begin{array}{l}\text { MANOVA de } \\
\text { Medidas } \\
\text { Repetidas }\end{array}$ & F Múltiple & $p$ & $\begin{array}{l}\text { MANOVA de } \\
\text { Medidas } \\
\text { Repetidas }\end{array}$ & F Múltiple & $p$ \\
\hline $\begin{array}{l}\text { Lambda de Wilks } \\
0.646 .\end{array}$ & $(3,38)=6.937$ & $\leq .001 . * * * *$ & $\begin{array}{l}\text { Lambda de Wilks } \\
0.718 .\end{array}$ & $(3,38)=4.970$ & $\leq .005 . * * *$ \\
\hline \multicolumn{3}{|c|}{$\begin{array}{l}\text { MANOVA Simple + Pruebas auditivas + Percibido } \\
\text { conocimiento del inglés del padre }\end{array}$} & \multicolumn{3}{|c|}{$\begin{array}{l}\text { MANOVA Simple + Pruebas escritas + Percibido } \\
\text { conocimiento del inglés del padre }\end{array}$} \\
\hline \multicolumn{3}{|l|}{ Efecto principal } & \multicolumn{3}{|l|}{ Efecto principal } \\
\hline $\begin{array}{l}\text { Lambda de Wilks } \\
0.620 \text {. }\end{array}$ & $(4,37)=5.672$ & $p \leq .001 . * * * *$ & $\begin{array}{l}\text { Lambda de Wilks } \\
0.709 \text {. }\end{array}$ & $(4,37)=3.795$ & $p \leq .011 .^{*}$ \\
\hline $\begin{array}{l}\text { Prueba auditiva } 1 \\
\text { (pre-test) }\end{array}$ & NS & & $\begin{array}{l}\text { Prueba escrita } 1 \\
\text { (pre-test) }\end{array}$ & NS & \\
\hline Prueba auditiva 2 & S & & Prueba escrita 2 & $\mathrm{~S}$ & \\
\hline Prueba auditiva 3 & $\mathrm{~S}$ & & Prueba escrita 3 & $\mathrm{~S}$ & \\
\hline Prueba auditiva 4 & $\mathrm{~S}$ & & Prueba escrita 4 & $\mathrm{~S}$ & \\
\hline
\end{tabular}

NS-No significativo

S-Significativo

* significativo al nivel .05.

** significativo al nivel .01.

$* * *$ significativo al nivel .005 .

$* * * *$ significativo al nivel .001 .

\subsection{Variable 2: Percibida ayuda del padre con el inglés (tabla 2)}

La variable de la Percibida ayuda dada por el padre mostró un efecto principal significativo menos veces sobre las pruebas auditivas que con la variable anterior: el Percibido conocimiento del inglés del padre. Sin embargo, hubo otros hallazgos reveladores. Así, el análisis de Medidas Repetidas MANOVA con las pruebas auditivas 1-4, y la variable de la 
Percibida ayuda dada por el padre, mostró un efecto principal. El MANOVA simple y la variable de la Percibida ayuda dada por el padre mostró un efecto principal significativo con las pruebas auditivas 1-4. Otro resultado significativo se encontró en una de las pruebas individuales (la segunda prueba auditiva) con un MANOVA simple. Para las pruebas escritas (también tabla 2) solo el MANOVA simple dio a conocer un efecto principal significativo únicamente en la tercera prueba escrita.

Tabla 2: Percibida ayuda dada por el padre: las estadísticas para las pruebas escritas y auditivas

\begin{tabular}{|c|c|c|c|c|c|c|}
\hline \multicolumn{3}{|c|}{$\begin{array}{l}\text { MANOVA de Medidas Repetidas en Pruebas } \\
\text { auditivas + Percibida ayuda dada por el padre }\end{array}$} & \multicolumn{4}{|c|}{$\begin{array}{l}\text { MANOVA de Medidas Repetidas en Pruebas } \\
\text { escritas + Percibida ayuda dada por el padre }\end{array}$} \\
\hline \multicolumn{3}{|c|}{ Efecto principal } & \multicolumn{4}{|c|}{ Efecto principal } \\
\hline & F Múltiple & $p$ & & \multicolumn{2}{|l|}{ F Múltiple } & $p$ \\
\hline $\begin{array}{l}\text { Lambda de Wilks } \\
0.792\end{array}$ & $(3,38)=3.317$ & $\leq .030 .^{*}$ & $\begin{array}{l}\text { Lambda de Wilks } \\
---\end{array}$ & \multicolumn{2}{|l|}{--- } & NS \\
\hline \multicolumn{3}{|c|}{$\begin{array}{l}\text { MANOVA Simple }+ \text { Pruebas auditivas + Percibida } \\
\text { ayuda dada por el padre }\end{array}$} & \multicolumn{4}{|c|}{$\begin{array}{l}\text { MANOVA Simple + Pruebas escritas + Percibida } \\
\text { ayuda dada por el padre }\end{array}$} \\
\hline \multicolumn{3}{|c|}{ Efecto principal } & \multicolumn{4}{|c|}{ Efecto principal } \\
\hline $\begin{array}{l}\text { Lambda de Wilks } \\
0.776\end{array}$ & $(4,37)=2.668$ & $\leq .047 .^{*}$ & Lambda de Wilks & NS & NS & \\
\hline $\begin{array}{l}\text { Prueba auditiva } 1 \\
\text { (pre-test) }\end{array}$ & \multicolumn{2}{|l|}{ NS } & \multicolumn{2}{|c|}{ Prueba escrita 1 (pre-test) } & \multicolumn{2}{|l|}{ NS } \\
\hline Prueba auditiva 2 & \multicolumn{2}{|c|}{$\begin{array}{l}\text { F Univariada }(1,40)=8.943 \\
p \leq .005 . * *\end{array}$} & \multicolumn{2}{|l|}{ Prueba escrita 2} & \multicolumn{2}{|l|}{ NS } \\
\hline Prueba auditiva 3 & \multicolumn{2}{|l|}{$\mathrm{NS}$} & \multicolumn{2}{|l|}{ Prueba escrita 3} & \multicolumn{2}{|c|}{$\begin{array}{l}\text { F Univariada }(1,40) \\
4.329 . \\
p \leq .044 .^{*}\end{array}$} \\
\hline Prueba auditiva 4 & \multicolumn{2}{|l|}{ NS } & \multicolumn{2}{|l|}{ Prueba escrita 4} & \multicolumn{2}{|l|}{$\mathrm{NS}$} \\
\hline
\end{tabular}

\subsection{Variable 3: Percibido número de veces ayudado por el padre (tabla 3)}

El análisis de Repetido de Medidas de MANOVA mostró un efecto principal significativo para el Percibido número de veces ayudado por el padre en ambas pruebas auditivas y escritas (tabla 3). Sin embargo, la tendencia en todas las pruebas, excepto la segunda, es negativa, es decir, más veces ayudado por el padre, notas más bajas saca el niño. Las pruebas escritas mostraron un efecto principal significativo. Un MANOVA simple no mostró ninguno efecto principal significativo en ninguna de las pruebas y, así, no figura en la tabla 3.

Tabla 3: El percibido número de veces ayudado por el padre con el inglés: las estadísticas para las pruebas escritas y auditivas

\begin{tabular}{|c|c|c|c|c|c|}
\hline \multicolumn{3}{|c|}{$\begin{array}{l}\text { MANOVA de Medidas Repetidas en Pruebas auditivas } \\
+ \text { Percibido número de veces ayudado por el padre }\end{array}$} & \multicolumn{3}{|c|}{$\begin{array}{l}\text { MANOVA de Medidas Repetidas en Pruebas } \\
\text { escritas + Percibido número de veces ayudado } \\
\text { por el padre }\end{array}$} \\
\hline \multicolumn{3}{|l|}{ Efecto principal } & \multicolumn{3}{|c|}{ Efecto principal } \\
\hline $\begin{array}{l}\text { (tendencia } \\
\text { negativa) }\end{array}$ & F Múltiple & $p$ & & F Múltiple & $p$ \\
\hline $\begin{array}{l}\text { Lambda de Wilks } \\
0.458\end{array}$ & $(15,94)=2.051$ & $\leq .019 .^{*}$ & $\begin{array}{l}\text { Lambda de } \\
\text { Wilks } 0.443\end{array}$ & $(15,94)=2.154$ & $\leq .013 .^{*}$ \\
\hline
\end{tabular}




\subsection{Variable 4: Percibido conocimiento del inglés de la madre (tabla 4)}

Para las pruebas auditivas, la tabla 4 muestra un efecto principal. Sin embargo, en la mayoría de los demás casos según las estadísticas estos no alcanzaron un nivel significativo. El análisis de Medidas Repetidas no mostró un efecto significativo tampoco y el MANOVA simple únicamente dio a conocer una $\mathrm{F}$ Univariada significativa en la segunda prueba auditiva.

Con las pruebas escritas el análisis de Medidas Repetidas, entre sujetos, sí mostró un efecto principal significativo. Un análisis de MANOVA simple con las pruebas escritas mostró un efecto principal significativo en la última prueba escrita solamente.

Tabla 4: Percibido conocimiento del inglés de la madre: las estadísticas para las pruebas escritas y auditivas

\begin{tabular}{|c|c|c|c|c|c|}
\hline \multicolumn{3}{|c|}{$\begin{array}{l}\text { MANOVA de Medidas Repetidas (en pruebas } \\
\text { auditivas) + Percibido conocimiento del inglés de la } \\
\text { madre }\end{array}$} & \multicolumn{3}{|c|}{$\begin{array}{l}\text { MANOVA de Medidas Repetidas (en pruebas } \\
\text { escritas) + Percibido conocimiento del inglés de la } \\
\text { madre }\end{array}$} \\
\hline \multicolumn{3}{|l|}{ Efecto principal } & \multicolumn{3}{|l|}{ Efecto principal } \\
\hline & F Múltiple & $p$ & & $\begin{array}{l}\text { F Univariada } \\
\text { (entre sujetos) }\end{array}$ & $p$ \\
\hline $\begin{array}{l}\text { Lambda de } \\
\text { Wilks }\end{array}$ & --- & NS & Entre sujetos & $(3,129)=3.760$ & $\leq .013 . *$ \\
\hline \multicolumn{3}{|c|}{$\begin{array}{l}\text { MANOVA Simple + pruebas auditivas + Percibido } \\
\text { conocimiento del inglés de la madre }\end{array}$} & \multicolumn{3}{|c|}{$\begin{array}{l}\text { MANOVA Simple + pruebas escritas + Percibido } \\
\text { conocimiento del inglés de la madre }\end{array}$} \\
\hline \multicolumn{3}{|l|}{ Efecto principal } & \multicolumn{3}{|l|}{ Efecto principal } \\
\hline \begin{tabular}{ll|} 
Lambda & $\mathrm{de}$ \\
Wilks 0.776 & \\
\end{tabular} & \begin{tabular}{|l|}
--- \\
I
\end{tabular} & NS & \begin{tabular}{|l|}
$\begin{array}{l}\text { Lambda de } \\
\text { Wilks }\end{array}$ \\
\end{tabular} & --- & NS \\
\hline $\begin{array}{l}\text { Prueba auditiva } 1 \\
\text { (pre-test) }\end{array}$ & \multicolumn{2}{|l|}{ NS } & Prueba escrita 1 & \multicolumn{2}{|l|}{ NS } \\
\hline Prueba auditiva 2 & \multicolumn{2}{|c|}{$\begin{array}{l}\text { Significativo; F Univariada }(1,40)= \\
7.610 . p \leq .009 . * *\end{array}$} & Prueba escrita 2 & \multicolumn{2}{|l|}{ NS } \\
\hline Prueba auditiva 3 & \multicolumn{2}{|l|}{ NS } & Prueba escrita 3 & \multicolumn{2}{|l|}{ NS } \\
\hline Prueba auditiva 4 & \multicolumn{2}{|l|}{ NS } & Prueba escrita 4 & \multicolumn{2}{|c|}{$\begin{array}{l}\text { Significativo: F Univariada }(1,40)= \\
\text { 5.271. } p<.027 . *\end{array}$} \\
\hline
\end{tabular}

\subsection{Variable 5: Percibida ayuda dada por la madre (ninguna tabla)}

Para las pruebas auditivas, ni las MANOVA de Medidas Repetidas, ni el análisis de MANOVA simple, mostraron un efecto principal significativo para la Percibida ayuda dada por la madre y las pruebas auditivas. Similarmente, para las pruebas escritas, la Percibida ayuda dada por la madre no dio un efecto principal significativo en ninguna.

\subsection{Variable 6: Percibido número de veces ayudado por la madre (ninguna tabla)}

Con las pruebas auditivas un análisis de Medidas Repetidas de MANOVA no mostró ningún efecto principal significativo en las pruebas auditivas. Aunque el análisis de MANOVA simple no mostró ninguna relación significativa tampoco, el punto notable es que la mayoría de las pruebas bajo este análisis estadístico mostraron una relación negativa. Con las pruebas escritas no se encontró ninguna relación significativa (se encontró) en los análisis. 


\section{Discusión y CONCLUSIONES}

A lo largo de esta investigación, se ha descubierto un conjunto específico de variables nuevo en este campo y asociadas al aprendizaje del inglés como idioma extranjero (EFL/ ILE). Las variables específicas del Percibido conocimiento del inglés de los padres, la Percibida ayuda dada por los padres y el Percibido número de veces ayudado por los padres no parece haberse investigado anteriormente. Además, los resultados obtenidos con el análisis estadístico fueron significativos en su gran mayoría.

En el estudio actual, el Percibido conocimiento de inglés del padre era altamente importante tanto en las pruebas auditivas como en las escritas. De esta manera, podemos confirmar la primera hipótesis formulada en esta investigación. Además, Elley (1994) encontró que la relación entre el nivel académico del padre y los logros en la escritura y lectura de sus hijos era directa y lineal. En el estudio de Olneck y Bills (1980) también los padres eran importantes. La influencia era significativa cuando tenían una educación universitaria. Gardner y Smythe (1975) encontraron que la motivación de los alumnos se reguló mediante la influencia paterna. Puede ser que el conocimiento del padre funcione de una manera similar e indirecta en el estudio original informado aquí.

Las estadísticas para la Percibida ayuda dada por el padre reflejaron una conexión con las pruebas auditivas y las pruebas escritas, pero especialmente sobre las primeras. Podemos confirmar la segunda hipótesis sobre la percibida ayuda del padre. El Percibido número de veces ayudado por el padre fue también significativo tanto en las pruebas escritas como en las pruebas auditivas, pero en las posteriores la tendencia era negativa. Parece ser que los padres dan más ayuda a los niños que tienen más dificultades a la hora de comprender el inglés.

El Percibido conocimiento del inglés de la madre fue significativo con las pruebas escritas. Podemos confirmar de forma parcial la primera hipótesis formulada con el percibido conocimiento de la madre. Con las pruebas auditivas, la segunda prueba se relacionaba significativamente con el Percibido conocimiento de la madre. La Percibida ayuda dada por la madre no mostró ninguno efecto significativo, pero la relación entre el Percibido número de veces ayudado por la madre, aunque no significativo, tuvo una tendencia negativa. Esto puede significar que también las madres ayudaron al niño que más lo necesitaba. Cano García (1995) detectó algo similar con niños de 10 años y alumnos de primaria de la misma nacionalidad que en nuestro estudio. En conclusión, se percibe que las madres en este estudio ayudaron a los hijos con su inglés escrito mientras por otro lado parece ser que el conocimiento del padre sirve como un tipo de motivación para adquirirlo de forma auditiva.

Hasta ahora, estas variables de los padres y madres no parecen haberse estudiado en nuestro campo de ILE. Quizás, se ha dado por hecho, debido a la fuerte relación encontrada con los padres y otros campos de la educación. Sin embargo, no es una situación que simplemente se debería ignorar o dar por hecho. Incluso, si las escuelas y las autoridades no están dispuestas a promover programas para incluir a los padres, hay cosas que los profesores pueden hacer para tener en cuenta este factor o falta de ayuda paterna y así ayudar a mejorar su propio contexto y el de sus alumnos. Por ejemplo, simplemente observando la situación y utilizando el cuestionario que ofrece este estudio. Este estudio ha descubierto preguntas con respecto al progreso verdadero de los alumnos, sobre cuánto están aprendiendo por mérito propio y cuánto se debe al conocimiento y ayuda de los padres. La importancia de esto está 
reflejada en las consecuencias que tiene, no solamente para estos últimos niños, sino especialmente para aquellos no receptores de ayuda alguna o conocimiento paterno.

Sin embargo, y mientras tanto, se puede obtener un cuadro más realista de la situación para el beneficio de todos. Además, este estudio aporta el cuestionario empleado, como una herramienta ya experimentada con éxito. Finalmente, animamos a los profesores a usar este cuestionario para recabar tanta información como sea posible acerca de sus alumnos. Sobre todo, a fin de asegurar notas justas para los alumnos desde la escuela primaria en adelante.

\section{REFERENCIAS}

Abbs, B., Worrall, A., \& Ward, A. (1993). Splash 1. Essex: Longman Group Ltd.

Benson, C.S. (1980). Time and how it is spent. Educational Finance and Organizational Research Perspectives for theFuture. Washington, DC: National Institute of Education (DHEW) Program on Educational Policy and Organisation. (ERIC Document Reproduction Services No. ED 188 318).

Burstall, C. (1970). French in the Primary School: Attitudes and achievement. London: National Foundation for Educational Research in England and Wales (NFER).

Burstall, C. (1975). "Factors affecting foreign language learning: A consideration of some recent research findings". Language Teaching and Linguistics: Abstracts, 8, 5-25.

Christenson, S.L. (1990). "Differences in students' home environments: The need to work with families". School Psychology Review, 19, 4: 505-517.

Cano García, F. (1995, August). Parental involvement in child's study and home learning. 6th European Conference for Research on Learning Instruction, Nijmegan, Holland.

Elley, W.B. (1994). The IEA Study of Literacy: Achievement and instruction in 32 school-systems. Oxford: Pergamon Press.

Gardner, R.C., \& Smythe, P.C. (1975). Second language acquisition: A social psychological approach. (Research Bulletin No. 332). University of Western Ontario, Department of Psychology. In R.Gardner (1985). Social Psychology and Second Language Learning: The role of attitude and motivation. London: Edward Arnold.

Grolnick, W.S., Ryan, R.M., \& Deci, E.L. (1991)."The inner resources for school achievement: Motivational mediators of children's perceptions of their parents". Journal of Educational Psychology, 83, 508-517.

Henderson, A.T. (Ed.) (1987). The Evidence Continues to Grow: Parent involvement improves student achievement. An annotated bibliography. Maryland: National Committee for Citizens in Education Special Report.

Longman Ltd. (1999) Zac's Wordgames. CD-ROM. (English-Spanish Bilingual version for home use). Essex: Author.

Madrid, D., \& Manrique, I. (1983) "Influencia de la familia en el lenguaje infantil". Apuntes de Educación, 13, abril-junio, 26-28.

Marjoribanks, K. (1984). “Occupational status, family environments and adolescents' aspirations: The Laosa Model”. Journal of Educational Psychology, 76, 4: 690-700.

Martin, M.O., Mullis, I.V.S., Gonzalez, E.J., \& Kennedy, A.M. (2003). Trends in Children's Reading Literacy Achievement 1991-2001: IEA's Repeat in Nine Countries of the 1991 Reading Literacy Study. Chestnut Hill, MA: Boston College. <<accessible online at HTTP://ISC.BC.EDU/ PIRLS2001_PUBS.HTML>> 
Mueller, C.W. \& Parcel, T.L. (1981). "Measures of socioeconomic status: Alternatives and recommendations". Child Development, 52, 1: 13-30.

Olneck, M.R., \& Bills, D.B. (1980). "What makes Sammy run? An empirical assessment of the Bowles-Gintis Correspondence Theory". American Journal of Education, 89, 1: 27-61.

Rio Heller, L., \& Fantuzzo, J.W. (1993). "Reciprocal peer tutoring and parent partnership: Does parent involvement make a difference?" School Psychology Review, 2, 3: 517-534.

Stevenson, D.L., \& Baker, D.P. (1987)." "The family school relation and the child's school performance". Child Development, 58, 1348-1357.

Keith, T.Z., Keith, P.B., Troutman, G.C., Bickley, P.G., Trivette, P.S., \& Singh, K. (1993). "Does parental involvement affect eighth grade student achievement? Structural analysis of national data". School Psychology Review, 22, 3: 474-496.

Vale, D. (1990). Early Bird 1. Activity-based English for children. Cambridge: Cambridge University Press.

West, A.W., Noden, P., \& Edge, A. (1998). "Parental involvement in education in and out of school". British Educational Research Journal, 24, 4: 461-484. 


\section{APÉNDICE 1 \\ CUESTIONARIO PARA NIÑOS DE PRIMARIA}

\section{Cuestionario}

Por favor, contesta las siguientes preguntas con tanto detalle como puedas. Si tienes alguna duda, pregunta al profesor/a la profesora quien estará muy dispuesto/a a ayudarte, si levantas la mano.

1) $\mathrm{Tu}$ nombre y apellidos

2) Tu dirección: $\mathrm{C} /$ número piso

ciudad/pueblo código postal número de teléfono

3) Edad

4) Fecha de nacimiento año mes día

5) Nacionalidad (por ejemplo: Española, Británica)

6) Profesión de tu padre

7) Lugar donde trabaja tu padre (la empresa y/o la calle)

8) Profesión de tu madre

9) Lugar donde trabaja tu madre

10) ¿Tu padre sabe inglés? Sí/No

11) ¿Tu madre sabe inglés? Sí/No

12) ¿Te ha ayudado tu padre con el inglés este año? Sí/No

13) ¿Cuántas veces?

14) ¿Te ha ayudado tu madre con el inglés este año? Sí/No

15) ¿Cuántas veces?

16) ¿Cuántos hermanas tienes?

17) ¿Cuántos hermanos tienes?

18) ¿Cuántos de tus hermanas saben inglés?

19) ¿Cuántos de tus hermanos saben inglés?

20) ¿Te han ayudado tus hermanas o hermanos con el inglés este año?

21) ¿Cuántas veces?

22) ¿Has repasado al inglés este año fuera de estas clases? Sí/No

23) ¿Con quien? 
24) ¿Cuántas veces?

25) ¿Has estudiado inglés antes? Sí/No

26) ¿A qué edad empezaste a estudiar inglés la primera vez? años y meses

27) ¿Has estudiado inglés en alguna academia? Sí/No

28) Nombre y dirección de la academia

29) ¿Tiempo allí? años meses

30) ¿Cuántas horas a la semana?....

31) Nombre y dirección de algunas otras academias donde has estudiado inglés:

32) ¿Tiempo allí? años meses

33) ¿Cuántas horas a la semana?

34) ¿Has estudiado inglés con un profesor particular en casa? Sí/No

35) ¿Cuánto tiempo? años meses

36) ¿Cuántas horas a la semana?....

37) ¿Cuáles son los nombres de los libros con que has estudiado inglés?

38) Color de la cubierta de los libros

39) Editoriales

40) ¿Otros materiales de inglés que has usado?

41) ¿Tienen pensados tus padres matricularte en clases particulares de inglés este curso? Sí/ No

42) ¿Te gustaría aprender inglés? Sí/No

43) ¿Te gusta asistir al colegio? Sí/No

44) ¿Has repasado al inglés este año fuera de estas clases? Sí/No

45) ¿Con quien?

46) ¿Cuántas veces?

47) ¿Cual es tu opinión de estas clases de inglés? Muy buenas/ buenas/no buenas ni malas/ no muy buenas/malas

48) ¿Sabias de antemano cuando íbamos a hacer exámenes?

49) ¿Has estado alguna vez en Gran Bretaña, USA, Australia u otro país donde hablan inglés? Sí/No

50) ¿Nombre del país tiempo allí meses años? 